\title{
Dipolar degrees of freedom and Isospin equilibration processes in Heavy Ion collisions.
}

\author{
M.Papa, ${ }^{1, *}$ I. Berceanu, ${ }^{2}$ L. Acosta,${ }^{3}$ F. Amorini, ${ }^{3}$ C. Agodi, ${ }^{3}$ A. Anzalone, ${ }^{3}$ \\ L. Auditore, ${ }^{4,5}$ G. Cardella, ${ }^{1}$ S. Cavallaro,${ }^{3}$ M.B. Chatterjee, ${ }^{6}$ E. De Filippo, ${ }^{1}$ \\ L. Francalanza, ${ }^{3,7}$ E. Geraci, ${ }^{1,8}$ L. Grassi, ${ }^{1,7}$ B. Gnoffo,,${ }^{1,7}$ J. Han ${ }^{3}$ E. La Guidara, ${ }^{1}$ \\ G. Lanzalone, ${ }^{3,9}$ I. Lombardo, ${ }^{10}$ C. Maiolino, ${ }^{3}$ T. Minniti, ${ }^{4}$ A. Pagano, ${ }^{1}$ E.V. Pagano, ${ }^{3,7}$ \\ S. Pirrone, ${ }^{1}$ G. Politi,${ }^{7}$ F. Porto,${ }^{3,7}$ L. Quattrocchi,${ }^{4}$ F. Rizzo,${ }^{3,7}$ E. Rosato,${ }^{11,10}$ \\ P. Russotto, ${ }^{3}$ A. Trifirò, ${ }^{4,5}$ M. Trimarchi,${ }^{5,11}$ G. Verde,${ }^{1}$ and M. Vigilante ${ }^{12}$ \\ ${ }^{1}$ INFN, Catania, Italy \\ ${ }^{2}$ National Institute for Physics, Bucharest, Romania \\ ${ }^{3}$ INFN-Laboratori Nazionali del Sud, Catania, Italy \\ ${ }^{4}$ INFN, Messina, Italy \\ ${ }^{5}$ Università facoltà di Fisica, Messina,Italy \\ ${ }^{6}$ Saha, Institute of Nuclear Physics Kolkata, India \\ ${ }^{7}$ Università facoltà di Fisica e Astronomia, Catania, Italy \\ ${ }^{8}$ Univeristà facoltà di Fisica e Astronomia, Catania, Italy \\ 9 "Kore" Università, Enna, Italy \\ ${ }^{10}$ Università facoltà di Fisica, Napoli, Italy \\ ${ }^{11}$ Universita' facolta di Fisica, Napoli, Italy \\ ${ }^{12}$ INFN, Napoli, Italy
}

(Dated: August 17, 2019) 


\begin{abstract}
Background: In heavy ion collision at the Fermi energies Isospin equilibration processes occurring when nuclei with different charge/mass asymmetries interacts have been investigated to get information on the nucleon-nucleon Iso-vectorial effective interaction.

Purpose: In this paper, for the system ${ }^{48} \mathrm{Ca}+{ }^{27} \mathrm{Al}$ at $40 \mathrm{MeV} /$ nucleon, we investigate on this process by means of an observable tightly linked to isospin equilibration processes and sensitive in exclusive way to the dynamical stage of the collision. From the comparison with dynamical model calculations we want also to obtain information on the Iso-vectorial effective microscopic interaction.
\end{abstract}

Method: The average time derivative of the total dipole associated to the relative motion of all emitted charged particles and fragments has been determined from the measured charges and velocities by using the $4 \pi$ multi-detector CHIMERA. The average has been determined for semiperipheral collisions and for different charges $Z_{b}$ of the biggest produced fragment. Experimental evidences collected for the systems ${ }^{27} \mathrm{Al}+{ }^{48} \mathrm{Ca}$ and ${ }^{27} \mathrm{Al}+{ }^{40} \mathrm{Ca}$ at $40 \mathrm{MeV} /$ nucleon used to support this novel method of investigation are also discussed.

Results: The data analysis shows a clear signature of a trend to the global Isospin equilibration of the system for increasing differences of the $Z_{b}$ values with respect to the projectile charge.

Conclusions: The comparison with CoMD-II calculations gives the best agreement with data using a stiffness $\gamma$ value for the Iso-vectorial interaction in the range $\gamma \simeq 1-1.2$. Moreover, the same comparison allows to estimate the non-negligible contribution to the global isospin equilibration process given by the un-detected emitted neutrons.

PACS numbers: 25.70.-z, 21.30.Fe

\footnotetext{
* papa@ct.infn.it
} 


\section{INTRODUCTION}

Experimental evidences on Heavy Ion Collisions highlight, in different aspects, processes evolving on different time scales. At the Fermi energies semiclassical dynamical models can not describe the system during its overall time evolution. According to the displayed phenomenology, the collected data are usually described as produced by a fast pre-equilibrium stage described through dynamical models [1-3] and later stage processes described by statistical decay models [4]. The statistical contribution is usually separated from the dynamical one through cut/extrapolation procedures applied to angular correlation and/or to particle kinetic energy spectra (see as an example [5, 6]). When clearly identified [7], the attempt to measure observables in principle closely linked to only one of the two regime is therefore highly desirable. In fact, this eventuality allows to decouple effects related to the two classes of mechanisms that are linked to rather different nuclear matter macroscopic properties. In the last decades great efforts have been performed to extract information on the nuclear iso-vectorial forces by studying charge/mass asymmetric systems [8-18]. These attempts concerns both the dynamical stage and the statistical decay of the produced hot sources. In particular in this last stage the isospin and excitation energy dependence of the level density formula play a key role and it is currently under investigation [10, 12, 18 20].

A phenomenon closely linked with iso-vectorial-forces is the well known process leading to the redistribution in phase-space of the charge/mass excess $\beta=\frac{N-Z}{A}[21]$ of the emitted particles and fragments ( $\mathrm{Z}$ is the charge, $\mathrm{N}$ is the neutron number and $\mathrm{A}$ the mass number). This phenomenon commonly referred as charge/mass or isospin equilibration process is rather complex in the Fermi energy domain, especially when finite size effects of the studied system have to be properly taken into account. The charge/mass distributions related to the final fragments and particles are affected by the pre-equilibrium stage, which includes particles and fragments production in the mid-rapidity region, prompt emission, transfer of mass and charge between the main fragments and finally particles/fragments emission from hot equilibrated sources through a multi-step statistical cascade. As an example, in Refs [11, 16, 21 23] the transfer of mass-charge between the main partners has been investigated through the study of the isospin transport ratio obtained starting from the isotopic distributions produced near the projectile rapidity for medium-heavy symmetric/quasy-symmetric systems. 
In this paper we report on results of investigations on this equilibration process for the system ${ }^{48} \mathrm{Ca}+{ }^{27} \mathrm{Al}$ at $40 \mathrm{MeV} /$ nucleon starting from a different and/or complementary point of view. The measurement was performed with the CHIMERA multi-detector [24] at Laboratori Nazionali del Sud di Catania (Italy). The main goal of the experiment was to evaluate, for well reconstructed events, belonging to selected classes $\mathcal{K}$, the quantity:

$$
\langle\vec{D}\rangle=\left\langle\sum_{i=1}^{m} Z_{i}\left(\vec{V}_{i}-\vec{V}_{c . m .}\right)\right\rangle_{\mathcal{K}} .
$$

The brackets indicate the average value over the ensemble $\mathcal{K} . Z_{i}, V_{i}, m$ are the charges, laboratory velocities, charged particle multiplicity respectively of the produced particles in the selected class of events, respectively. Finally $\vec{V}_{c . m}$ is the center of mass (c.m.) velocity. We note that in this expression the contribution of produced neutral particles is implicitly contained in $\vec{V}_{c . m}$. The interest on this quantity was triggered by two main reasons:

a) as shown in Refs [7, 25] this quantity is closely linked with charge/mass equilibration process because it represents the average time derivative of the total dipolar signal in the asymptotic stage (expressed in unit of $e$ ). In fact, as an example, for binary systems, in absence of dynamical neutron-proton collective motion we have: $\langle\vec{D}\rangle \equiv \vec{D}_{m}=\frac{1}{2}\langle\mu\rangle\left(\left\langle\beta_{2}\right\rangle-\right.$ $\left.\left\langle\beta_{1}\right\rangle\right)\left(\left\langle\vec{V}_{1}-\vec{V}_{2}\right)\right\rangle$. $\mu$ is the reduced mass number of the system, $\beta_{1}, \beta_{2}$ are the isospin asymmetries of the two partners 1 and 2 and finally $\vec{V}_{1}$ and $\vec{V}_{2}$ the related velocities. In the above expression we have supposed negligible the correlation of fluctuations between charge/mass ratios of the partners and their relative velocity. On the other limit, the same quantity is zero if evaluated for a system represented by an equilibrated source before or after the statistical decay. As shown from dynamical microscopic calculations in a collision process between two nuclei having different charge/mass asymmetries, $|\langle\vec{D}\rangle|$ changes during the time towards smaller values in the pre-equilibrium stage (spontaneous approach to the equilibrium) producing $\gamma$-ray emission through the excitation of a more or less damped dipolar dynamical mode [7, 26];

b) because of the symmetries of the statistical decay mode, $\langle\vec{D}\rangle$ is not affected by the statistical emission of all the produced sources in later stages, as it is shown in Ref. [7]. This essentially happens because, due to the vectorial kinematical character of this quantity , for well reconstructed events statistical effects are self-averaged to zero. Therefore $\langle\vec{D}\rangle$ is a rather well suited global variable to selectively evidence dynamical effects related to the Isospin equilibration process. 


\section{THE EXPERIMENTAL PROCEDURE AND DATA ANALYSIS}

The experiment was carried out by using $40 \mathrm{MeV} /$ nucleon ${ }^{48} \mathrm{Ca}$ and ${ }^{27} \mathrm{Al}$ beams, at the LNS Super-Conducting Cyclotron. The beam impinged on $400 \frac{\mu \mathrm{g}}{\mathrm{cm}^{2}}{ }^{27} \mathrm{Al}$ and about $1200 \frac{\mu \mathrm{g}}{\mathrm{cm}^{2}}$ ${ }^{48} \mathrm{Ca},{ }^{40} \mathrm{Ca}$ targets. The chosen combinations were the following: ${ }^{48} \mathrm{Ca}+{ }^{27} \mathrm{Al}$ as the main system to be investigated, the ${ }^{27} \mathrm{Al}+{ }^{48} \mathrm{Ca}$ as the auxiliary system (see the following) and the charge/mass quasi-symmetric ${ }^{27} \mathrm{Al}+{ }^{40} \mathrm{Ca}$ as reference system. Charges, masses, energies and velocities of the produced particles and fragments were measured with the $4 \pi$ Multi-Detector CHIMERA [24, 27]. In particular, the $\Delta E-E$ technique was employed for Z identification of fragments punching through the silicon detectors and additionally for isotopic identification of fragments with atomic numbers $Z<10$. Mass identification is performed with the timeof-flight (TOF) technique by using the time signal from silicon detectors with respect to the time reference of the radio-frequency signal from the cyclotron. The TOF technique is basically used for velocity measurements of heavy ions. This technique is also essential for the mass and charge indirect determination of slow TLF's (Target Like Fragments) stopped in the silicon detectors. Energetic light charged particles, stopped in the scintillator crystal, are identified by applying the "fast-slow" discrimination method [28].

In the following we report results collected for events with a multiplicity of detected charged particles greater than or equal to 2. We have chosen rather restrictive selection criteria to identify the "good" reconstructed events. These conditions are suggested from calculations to obtain the "invariance" of the investigated quantity with respect the statistical decay mode (see Table I). For the main system we have selected events for which the total identified charge $Z_{t o t}^{d}=33$. Checks are being made to see at what extent the previous condition can be slightly released always keeping the above mentioned "invariance" condition to an acceptable level. The total detected mass was chosen in the interval $62 \leq A_{\text {tot }}^{d} \leq 78$. The total measured momentum along the beam axis has been selected within $70 \%$ of the theoretical value $(422 \mathrm{amu} \cdot \mathrm{cm} / \mathrm{nsec})$.

Analogous conditions have been imposed for the others investigated systems, taking into account the differences in the total mass and c.m. velocity. The well reconstructed events have been classified according to the charge of the biggest detected fragment $Z_{b}$ and according to the estimated total kinetic energy loss $T K E L=\mu E_{A}^{i n}-\sum_{i=1}^{m} \frac{1}{2}\left(M_{i} V_{i}^{2}-M_{t o t}^{d} V_{c . m . d}^{2}\right)$. Where $E_{A}^{i n}$ is the incident energy per nucleon and $\mu$ is reduced mass number of the impinging 


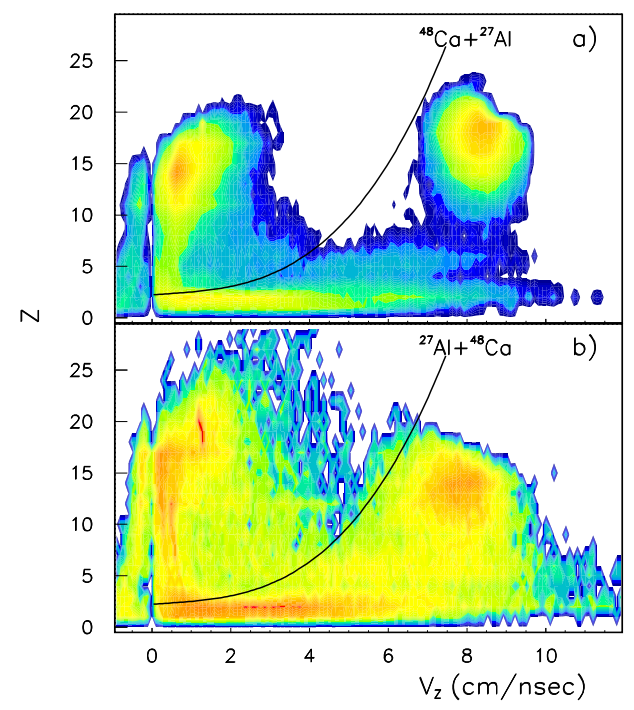

FIG. 1. (Color online) Charges $\mathrm{Z}$ of the detected fragments versus their velocities $V_{Z}$ along the beam axis are shown for the main system (panel a)) and the auxiliary one (panel b).

nuclei. In the above expression $M_{i}, V_{i}$ are the measured masses and velocities; $M_{\text {tot }}^{d}$ is the total measured mass and $\vec{V}_{c . m ., d}$ the related c.m. velocity associated to the detected charged particles. Therefore, each event has been characterized also through these two last quantities, that in the TKEL evaluation can globally compensate the uncertainties due to the non perfect mass identification and velocity measurement. For the main system ${ }^{48} \mathrm{Ca}+{ }^{27} \mathrm{Al}$ and the auxiliary one ${ }^{27} \mathrm{Al}+{ }^{48} \mathrm{Ca}$, in Fig.1 we show the charge of the detected fragments as function of their velocity $V_{Z}$ along the beam direction. In both cases the bi-dimensional plots show the dominance of processes producing TLF's and PLF's (Projectile Like fragments). The thin black curves represent the threshold of the $\Delta E-E$ charge identification technique as function of the fragments velocity evaluated for the typical Silicon detector thickness of the CHIMERA apparatus (about $300 \mu \mathrm{m}$ ).

For each velocity value, fragments having a charge smaller than the value plotted through the curves can be directly assigned by means of the $\Delta E-E$ technique. Fragments having an higher charge will be stopped in the silicon detector an then the charge identification is obtained in an indirect way through the mass determination obtained by means of TOF and energy measurements and by using the Charity prescription [29]. 


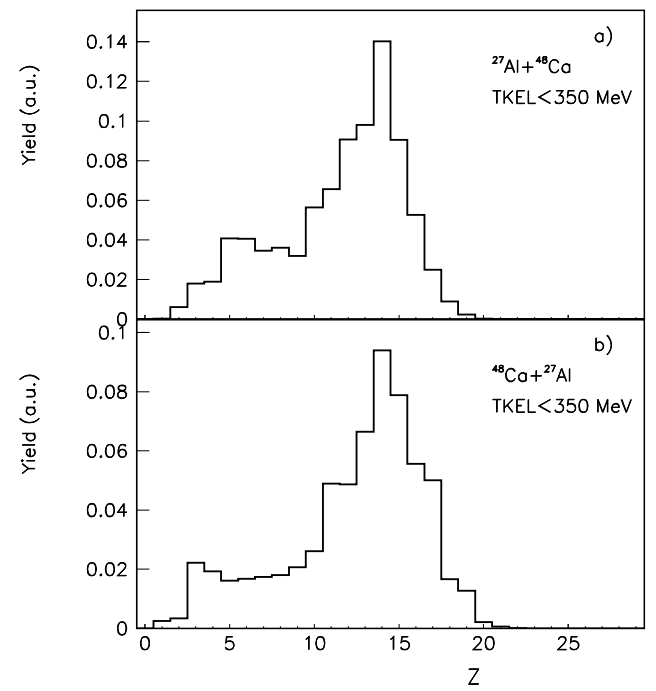

FIG. 2. For TKEL $<350 \mathrm{MeV}$, (panel a): Charge distribution of PLF's punching through the Si detectors (direct estimate based on the $\Delta E-E$ method) for the ${ }^{27} \mathrm{Al}+{ }^{48} \mathrm{Ca}$ system at 40 $\mathrm{MeV} /$ nucleon (panel b): Charge distribution of TLF's stopped in the $S i$ detectors (indirect estimate based on the TOF method) for the ${ }^{48} \mathrm{Ca}+{ }^{27} \mathrm{Al}$ system at $40 \mathrm{MeV} /$ nucleon

Therefore the comparison between the TLF charge distribution obtained in the main system in the indirect way (upper portion of the Z- $V_{Z}$ plot in Fig.1(a)) and the one related to the PLF for the auxiliary system, directly assigned, (lower portion of Z- $V_{Z}$ plot in Fig.1(b)) allows to evidence eventual systematic errors in the indirect charge assignment associated to the main system. The comparison has been performed for different windows of TKEL.

It results that for TKEL lower than about $350 \mathrm{MeV}$ the obtained charge distributions are similar and no systematic error ${ }^{0}$ is evidenced. This ${ }^{2}$ resule is shown in Fig.2 in panels a) and b) for the main system and the auxiliar one. For larger value of TKEL, the two distributions are different. In fact in this case due to the opposite kinematical conditions and to the finite geometrical efficiency of the detector, different reaction mechanisms are selected on average and the comparison is difficult.

The present study is focused on the investigation of the behavior of the component of $\vec{D}$ along the beam axis $Z, D_{Z}$. As for the case of the TKEL determination and especially to compensate the eventual systematic errors in the fragment velocity determination, instead of the theoretical value $\vec{V}_{c . m}$., we are necessarily induced to use in eq.(1) the value of 


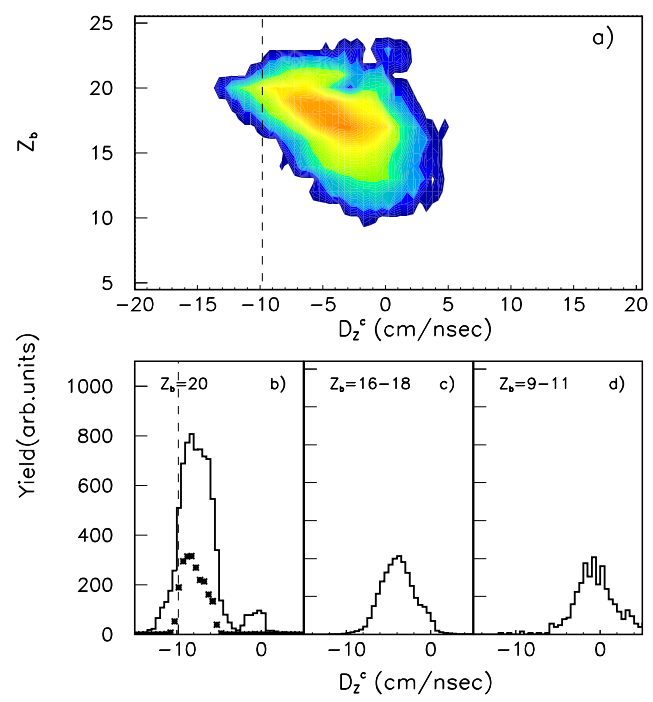

FIG. 3. (Color online) (panel a): For the system ${ }^{48} \mathrm{Ca}+{ }^{27} \mathrm{Al}$ at $40 \mathrm{MeV} /$ nucleon the measured values of $D_{Z}^{c}$ are plotted for different $Z_{b}$ associated to the selected events (charged multiplicity $m \geq 2$ ). The dot-dashed vertical lines indicates the reference limiting values $D_{m}$ (see the text). (panels b),c),d)): $D_{Z}^{c}$ distributions obtained as projection of the above bi-dimensional plot for different $Z_{b}$ intervals. In the panel b) the $D_{Z}^{c}$ spectrum for $Z_{b}=20$ and for quasi-elastic events TKEL $<70 \mathrm{MeV}$ is plotted with star symbols.

$V_{c . m ., d}^{Z}$ evaluated, event by event, from the velocities along the beam axis of all the detected charged particles. The obtained quantity will be named in the following $D_{Z}^{c}$. $D_{Z}^{c}$ therefore represents a partial dipolar signal related to the intrinsic motion of the subsystem formed by all the produced charged particle. The contribution related to the global relative motion of the undetected free neutrons is not included. In the next sections we will describe a way to estimate the average total signal associated to $D_{Z}$. In Fig.3 (panel a)), for the main system, we show the correlation plot $Z_{b}$.v.s. $D_{Z}^{c}$ for the selected events. The ridge in the plot highlights an increasing trend of $\left\langle D_{Z}^{c}\right\rangle$ going from negative values to almost zero for decreasing values of $Z_{b}$ respect to $Z_{P L F}$. We note that according to the expression given for $D_{m}$, in the initial configuration the system should exhibit a limiting value of $\left\langle D_{Z}^{c}\right\rangle$ (grazing collisions) close to about $-9.8 \mathrm{~cm} / \mathrm{nsec}$. The increasing average values of $\left\langle D_{Z}^{c}\right\rangle$ for $Z_{b}$ different from projectile atomic number (less peripheral collision), represents a clear signature of the evolution through charge/mass equilibration values (see next section). The limiting value 
corresponding to almost "grazing" collisions (along the beam axis), is evidenced in Fig.3 by dot-dashed vertical lines. Panels b),c) and d) show the projections of the bi-dimensional plot for different intervals of $Z_{b}$ and we can clearly see the trend of $\left\langle D_{Z}^{c}\right\rangle$. In particular, in panel b) the spectrum with star symbols is obtained by imposing a value of TKEL $<70$ $\mathrm{MeV}$ typical for quasi-elastic processes. The large fluctuations of $D_{Z}^{c}$ around the average value are due to physical reasons (the particular "history" of the each event) and to the measurement procedure reflecting the related uncertainties. In Fig.4 we show analogous plots for the reference isospin quasi-symmetric system ${ }^{27} \mathrm{Al}+{ }^{40} \mathrm{Ca}$ at $40 \mathrm{MeV} /$ nucleon. In this case the limiting value for "grazing" collision, $D_{m}$ is about $-2.6 \mathrm{~cm} / \mathrm{nsec}$ and, how can be clearly seen, the experimental plots shows values close to zero and an enhancement near the $D_{m}$ value (Fig.4b). The check on this system as compared to the main one ensure us on the good level of confidence obtained in the determination of $\left\langle D_{Z}^{c}\right\rangle$.

\section{COMPARISON WITH COMD-II CALCULATIONS}

To obtain information on the behavior of the Iso-vectorial interaction starting from the present experimental data, we have performed CoMD-II [30, 31] calculations in the interval of impact parameters $b=4-9 \mathrm{fm}$ (the weight of each $b$ is chosen proportionally to $b$ itself). Dynamical calculations have been followed up to about $500 \mathrm{fm} / \mathrm{c}$. After this primary stage, the produced hot main sources have on average an excitation energy lower than 2.5 $\mathrm{MeV} /$ nucelon. For each generated event, a second stage of a multi-step statistical decay has been simulated through the Monte Carlo GEMINI code [32]. The obtained results have been analyzed with an implemented code that takes into account the main filtering effects of the experimental apparatus. This include geometrical acceptance of the identified particles and the main selection criteria used in the analysis of experimental data.

In particular the CoMD-II calculations have been performed with stiffness parameter values concerning the Iso-vectorial interaction $\gamma=0.5, \gamma=0.8, \gamma=1, \gamma=1.2, \gamma=1.5$ and symmetry energy about $32 \mathrm{MeV}$. According to previously performed investigations, the parameters of the effective Skyrme interaction corresponding to a compressibility of about $220 \mathrm{MeV}$ have been chosen following Ref.[33]. In Fig.5 (panel A),B) C)) we show the com-

parison of the calculations with the measured value of $\left\langle D_{Z}^{c}\right\rangle$ (red points) evaluated starting from the already shown correlation plot in Fig.3. The values are referred to different $Z_{b}$ 

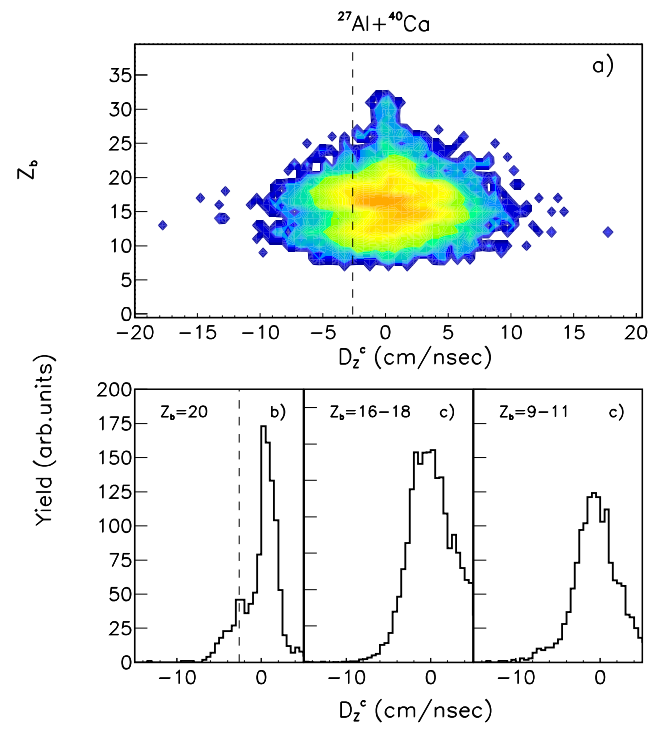

FIG. 4. (Color online) Same as the Fig.2 but for the reference system ${ }^{27} \mathrm{Al}+{ }^{40} \mathrm{Ca}$

windows and TKEL $<350 \mathrm{MeV}$. The vertical bars indicate the evaluated statistical errors which include also, conservatively, a non-systematic uncertainty on the measured fragment charge $\Delta Z= \pm 1$. The corresponding theoretical values are plotted in the different panels with black symbols. The obtained values for $\gamma=0.5$ are rather different from the experimental results, they are out of the range value plotted in Fig.5. For this case a maximum value about $7 \mathrm{~cm} / \mathrm{nsec}$ is obtained for $Z_{b}=9-11$ while the minimum value about -20 $\mathrm{cm} / \mathrm{nsec}$ is obtained for $Z_{b}=21-22$. The comparison of the others calculations with the experimental data is quantified in the figure through the shown $\chi^{2}$ value. According to this parameter, the case with $\gamma=1$ gives the best comparison even if it is only slightly better than the case $\gamma=1.2$. The behavior of $\left\langle D_{Z}^{c}\right\rangle$ (related to only charged fragments and particles) can be understood in a qualitative way according to the following considerations: as shown in the previous figure (apart from the case $\gamma=0.8$ ) for $Z_{b}$ smaller than $Z_{P L F}$ (dominance of mass transfer from PLF to TLF) and for $Z_{b}$ larger than $Z_{P L F}$ (on-set of fusion,incomplete fusion) $\left\langle D_{Z}^{c}\right\rangle$ increases (there are on average more charge/mass symmetric produced fragments). In fact,in this case it can be verified that $\left\langle V_{c . m ., d}^{Z}\right\rangle \leq V_{c . m}$. therefore the c.m. of the free neutrons has to be necessarily larger than $V_{c . m \text {. }}$, that means, on average, more neutron emission from the PLF side. The case of $\gamma=0.8$ produce instead for $Z_{b}>Z_{P L F}$ a larger negative value $\left\langle D_{Z}^{c}\right\rangle$. In this case $\left\langle V_{c . m ., d}^{Z}\right\rangle \geq V_{c . m}$. and therefore the cloud 


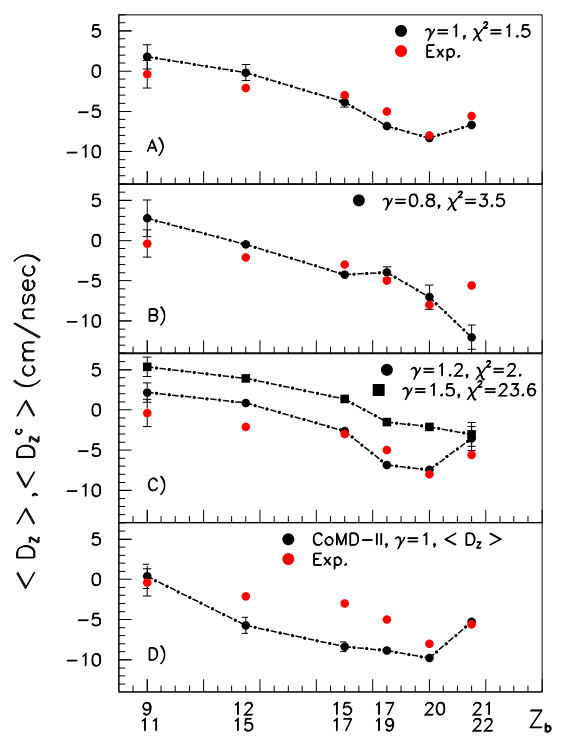

FIG. 5. (Color) For the investigated system ${ }^{48} \mathrm{Ca}+{ }^{27} \mathrm{Al}$ we plot for different $Z_{b}$ windows (the related extremes are indicated by the double labeling in the $Z_{b}$ axis) and for TKEL $<350 \mathrm{MeV}$ the measured average dipolar signal $\left\langle D_{Z}^{c}\right\rangle$ (red point). The experimental value are compared in the same figure with the results of COMD-II + GEMINI calculations (black point) filtered through the simulated response of the experimental apparatus and data-analysis selections. Panel A),B),C) show the comparisons for different $\gamma$ values characterizing the iso-vectorial interaction. The $\chi^{2}$ values with respect to the experimental data are also shown. Panel D): the calculated values of $\left\langle D_{Z}\right\rangle$ for $\gamma=1$ are compared to the experimental values for $\left\langle D_{Z}^{c}\right\rangle$. The error bars represent uncertainties due to the statistics of the simulations and measurement. The estimated uncertainties related to experimental values $\left\langle D_{Z}^{c}\right\rangle$ are in many cases smaller than the plotted symbols.

of neutrons has a c.m. velocity smaller than $V_{c . m .}$. In this case we have on average more neutron emission from the mid-rapidity and TLF side. A closer look to the fragments put in evidence in this case a dominance of processes producing a PLF fragment, only partially equilibrated in charge/mass and a almost complete disassembly of the TLF. Up to now we have discussed the behavior of $\left\langle D_{Z}^{c}\right\rangle$ which shows a good sensitivity to the parameters of the effective interaction. To recover information on the global degree of isospin equilibration we can evaluate $\left\langle D_{Z}\right\rangle$ through calculations by using the same set of parameters which "best fit" the experimental value of $\left\langle D_{Z}^{c}\right\rangle$. In Fig.5 (panel D)), we compare the calculated values of $\left\langle D_{Z}\right\rangle$ for $\gamma=1$ with CoMD-II+GEMINI (including the efficiency effect) with the exper- 
imental values $\left\langle D_{Z}^{c}\right\rangle$. The connection between the two quantities can be approximated by the following simple relation: $\left\langle D_{Z}\right\rangle \cong\left\langle D_{Z}^{c}\right\rangle+Z_{\text {tot }}^{d}\left(\left\langle V_{c . m .,}^{Z}\right\rangle-V_{c . m .}\right)$ where $\left\langle V_{c . m ., c}^{Z}\right\rangle$ is the c.m. velocity for the subsystem of the charged particles. Therefore the second term in the above expression give us an estimation of the effect associated to the undetected free neutrons [34] which clearly participate in to determining the global degree of isospin equilibration. Before to conclude this section we add some observations about the "invariance" of the discussed quantities with respect statistical decay modes. As previously observed this was shown in a rather general way in ref. [7]. In the present work we have also checked if this insensitivity is still maintained by operating with a realistic efficiency of the CHIMERA apparatus. In particular, in the "simulated" analysis we can keep the memory of the selected primary events from the dynamical model ordered according to the different $Z_{b}$ values and TKEL. It is therefore possible to evaluate the same quantity from the dynamical model $\left\langle D_{Z}^{D}\right\rangle$ without taking into account the GEMINI secondary decay processes and comparing it to the value of $\left\langle D_{Z}\right\rangle$ obtained from the complete calculations including the efficiency effects. The calculations have been performed for different $Z_{b}$ windows and for $T K E L<350 \mathrm{MeV}$. Table I, as an example, collects the obtained results for different $Z_{b}$ bins by using the calculations for $\gamma=1$. From the table we can appreciate the close correspondence between the results

TABLE I. For different windows of $Z_{b}$ and for TKEL $<350 \mathrm{MeV}$ the values $(\mathrm{cm} / \mathrm{nsec})$ of $\left\langle D_{Z}\right\rangle$ and the corresponding values of $\left\langle D_{Z}^{D}\right\rangle$ are shown for the ${ }^{48} \mathrm{Ca}+{ }^{27} \mathrm{Al}$ system for $\gamma=1$

\begin{tabular}{ccc}
\hline$Z_{b}$ & $\left\langle D_{Z}\right\rangle(\mathrm{cm} / \mathrm{nsec})$ & $\left\langle D_{Z}^{D}\right\rangle(\mathrm{cm} / \mathrm{nsec})$ \\
\hline $12-15$ & -5.73 & -5.9 \\
$15-17$ & -8.36 & -8.34 \\
$17-19$ & -8.86 & -8.87 \\
20 & -9.79 & -9.78 \\
$21-22$ & -5.36 & -5.30 \\
\hline
\end{tabular}

after the GEMINI [32] de-excitation stage and the ones related to the dynamical primary events. 


\section{SUMMARY AND OUTLOOKS}

In summary, dipolar degrees of freedom for the collision ${ }^{48} \mathrm{Ca}+{ }^{27} \mathrm{Al}$ at $40 \mathrm{MeV} /$ nucleon have been investigated for the first time by means of $4 \pi$ multi-detector CHIMERA. The study has been carried out through the measurement of the observable $\left\langle D_{Z}^{c}\right\rangle$ (associated to all the emitted charged particles) and through a related estimation of the $\left\langle D_{Z}\right\rangle$ values which are closely linked with the global Isospin equilibration process along the beam direction. The discussed insensitivity to the statistical decay make this observable able to investigate in an exclusive manner the overall dynamics on this phenomenon. The study performed on the behavior of $\left\langle D_{Z}^{c}\right\rangle$ and $\left\langle D_{Z}\right\rangle$ as function of the size of the biggest fragments for TKEL $<350$ $\mathrm{MeV}$ shows a noticeable sensitivity of this observable to the parameters of the microscopic effective interaction. First attempts to reproduce the experimental values of $\left\langle D_{Z}^{c}\right\rangle$ in essentially binary processes show an overall agrement with CoMD-II calculations using a stiffness parameter for the iso-vectorial interaction $\gamma \simeq 1 \div 1.2$. More experimental investigation should involve different systems and different reaction mechanisms. In particular, a next step forward in this kind of measurements would require a more detailed investigation including a reliable valuation and/or minimizations of possible systematic errors on the velocity of the produced charged particles. This would permit a reliable experimental estimation of $\left\langle D_{Z}\right\rangle$ allowing also for a corresponding experimental valuation of the global effect associ-

ated to the dynamically emitted neutrons. For this purpose long measurements involving targets and projectiles having the same charge/mass asymmetry (vanishing values of $\left\langle D_{Z}\right\rangle$ independently from the reaction mechanism) are necessary. A more detailed analysis could also allows to estimate the specific contribution to the equilibration process produced by the prompt and mid-rapidity emission as compared to the transfer of charge/mass between the main partners. The more challenging attempt to investigate on the other component along the $\mathrm{X}$ impact parameter direction could be of relevant interest. $\left\langle D_{X}\right\rangle$ in fact is linked, in a global way, and in an independent way from statistical emission, with the differential flow of particles characterized by different charge/mass asymmetries values [35].

[1] Bao-An Li, Lie-Wen Chen, Che Ming Ko, Phys. Rep. 464, 113(2008) and reference therin.

[2] V.Baran, M.Colonna, V.Greco, M. Di Toro, Phys. Rep. 410, 335(2005) and reference therin. 
[3] A.Bonasera, F.Gulminelli, and J.Molitoris, Phys. Rep. 243, 1(1994).

[4] J.B.Bondorf, A.S.Botvina, A.S. Lljinov, I.N. Mishustin, K.Sneppen, Phys. Rep. 257, $133(1995)$

[5] S.Piantelli et al., Phys. Rev. C. 78, 064605 (2008).

[6] P.Russotto et al., Phys. Rev. C. 81, 064605 (2010).

[7] M.Papa et al., Phys. Rev. C 72,064608(2005) and reference therein (see in particular Appendix D).

[8] P. Danielewicz and J.Lee, Nucl.Phys A922 1(2014).

[9] G.Ademard et al., Eur. Phys. Jour. A 50 33(2014).

[10] G.Ademard et al., Phys. Rev. C. 83, 054619 (2011).

[11] M.B.Tsang et al.,Phys. Rev. Lett. 92, 062701 (2004) and reference therein.

[12] P.Marini et al., Phys. Rev. C. 87, 024603 (2013).

[13] Z.Kohley et al., Phys. Rev. C. 85, 064605 (2012).

[14] F.Amorini et al, Phys. Rev. Lett. 102, 112701(2009).

[15] G.Cardella et al.,Phys. Rev. C. 85, 064609 (2012) and reference therein.

[16] Z.Chen et al.,Phys. Rev. C. 81, 064613 (2010).

[17] K.Brown, S.Hudan, R.T. deSouza, J.Gauthier, R.Roy, D.V.Shetty, G.A.Souliotis, S.J.Yennello, Phys. Rev. C. 87, 061601 (2013).

[18] P.Marini, M.F.Rivet, B.Borderie, N.Le Neindre, A.Chnihi, G.Verde and J.P.Wieleczko, EPJ Web of Conferences 2, 04003(2010).

[19] A.Brondi et al.,EPJ Web of Conferences 2, 04002(2010).

[20] S.Pirrone et al.,EPJ Web of Conferences 17, 16010(2011).

[21] F.Rami et al.,Phys. Rev. Lett. 84, 1120(2000).

[22] I.Lombardo et al.,Phys. Rev. C. 82, 014608 (2010).

[23] Z.Y.Sun et al.,Phys. Rev. C. 82, 051603 (2010).

[24] A.Pagano et al.,Nucl.Phys A734 504(2004);A.PAgano, Nucl.Phys.NEws 22 25(2012).

[25] G.Giuliani and M.Papa, Phys. Rev. C 73,031601(2006).

[26] F.Amorini et al., Phys. Rev C 58, 987(1998).

[27] E.De.Filippo et al., Phys. Rev. C 71, 044602(2005).

[28] M.Alderighi et al., Nucl. Instr. Meth. A489, 257(2002).

[29] R.J.Charity, Phys. Rev C 58, 1073(1998) . 
[30] M. Papa, T. Maruyama and A. Bonasera, Phys. Rev. C 64 024612(2001).

[31] M.Papa, G.Giuliani A.Bonasera; Jou. of Comp. Physics 208,403(2005).

[32] R.J. Charity, D.R. Bowman and Z.H. Liu, R.J. McDonald, M.A. McMahan, G.J. Wozniak, L.G. Moretto, S. Bradley, W.L. Kehoe, and A.C. Mignerey, Nucl. Phys. A476, 516 (1988).

[33] M.Papa, Phys. Rev C 87, 014001(2013).

[34] Z.Kohley et al., Phys. Rev C 88, 041601(2013).

[35] M.Papa M and G.Giuliani, Journal of Physics: Conference Series 312 082034(2011). 